\title{
Corrigendum
}

\section{Corrigendum to "Performance Evaluation of Crumb Rubber Asphalt Modified with Silicone-Based Warm Mix Additives"}

\author{
Nonde Lushinga $\left(\mathbb{D},{ }^{1,2}\right.$ Liping Cao ${ }^{1},{ }^{1}$ Zejiao Dong, ${ }^{1}$ Chen Yang, ${ }^{1}$ and Cyriaque O. Assogba ${ }^{1}$ \\ ${ }^{1}$ School of Transportation Science and Engineering, Harbin Institute of Technology, Harbin 150090, China \\ ${ }^{2}$ School of Built Environment, The Copperbelt University, Kitwe 10101, Zambia \\ Correspondence should be addressed to Liping Cao; caoliping79@163.com
}

Received 22 May 2021; Accepted 22 May 2021; Published 28 July 2021

Copyright (c) 2021 Nonde Lushinga et al. This is an open access article distributed under the Creative Commons Attribution License, which permits unrestricted use, distribution, and reproduction in any medium, provided the original work is properly cited.

In the article titled "Performance Evaluation of Crumb Rubber Asphalt Modified with Silicone-Based Warm Mix Additives" [1], the authors identified that there were errors in Figures 3, 6, and 9.

In Figure 3, "low temperature" should be changed to "high temperature." In Figure 6, the decimal points had been omitted in error and are added in the revised version below.
In Figure 9(a), the authors identified the control and XP data appeared to be the same due to an error during the preparation of the manuscript and the correct values are provided in the updated figure below.

The authors confirm that these errors do not affect the conclusions of the article and the corrected Figures are as follows.

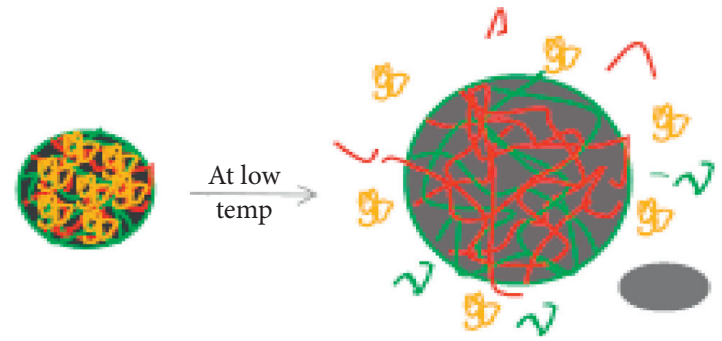

(a)

(b)

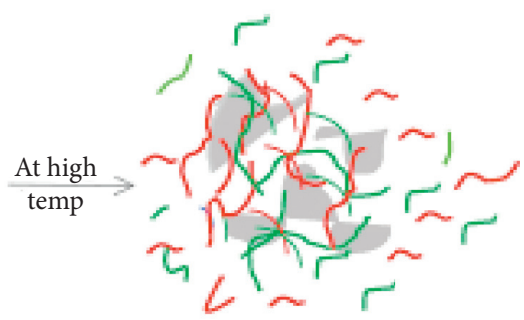

(c)

Natural rubber

Synthetic rubber

Oil

Fillers and carbon black

FIGURE 3: CR swelling and degradation in asphalt [36]. (a) Crumb rubber (CR). (b) Swollen CR, discharging oil and fillers to asphalt. (c) Breaking of CR. 


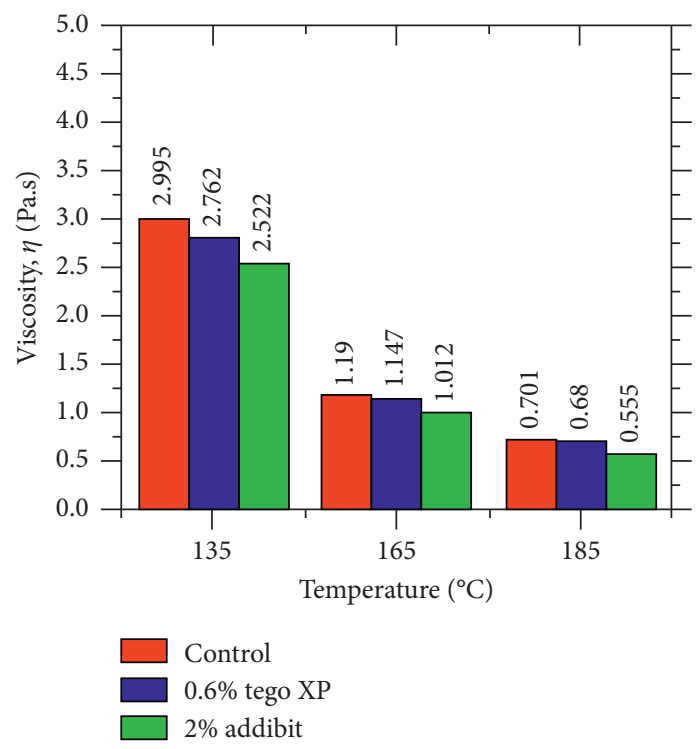

FIGURE 6: Viscosity and shear rate of CRM asphalt with and without WMA additives at (d) viscosity-temperature at 1000 shearing rate (1/s).

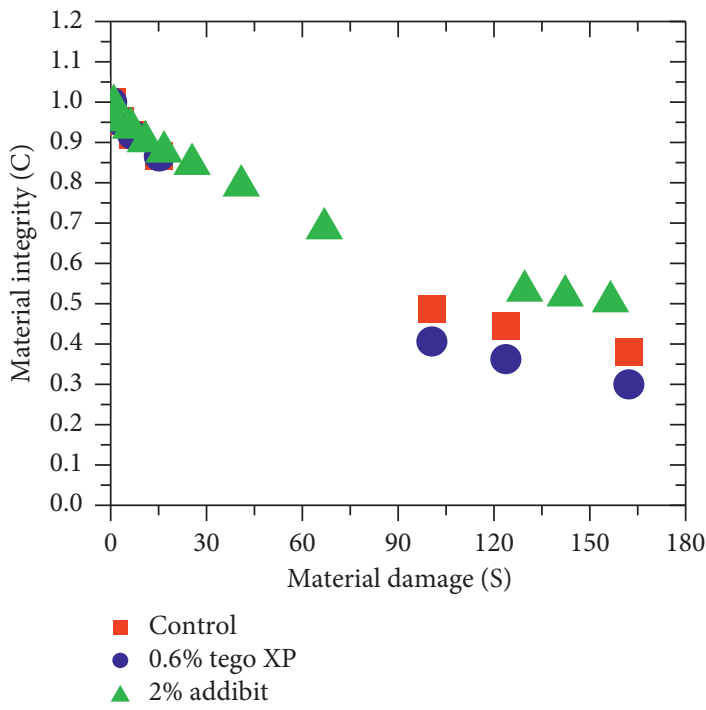

FIgURE 9: (a) VECD damage curve.

\section{References}

[1] N. Lushinga, L. Cao, Z. Dong, C. Yang, and C. O. Assogba, "Performance Evaluation of Crumb Rubber Asphalt Modified with Silicone-Based Warm Mix Additives," Advances in Civil Engineering, vol. 2020, Article ID 4840825, 17 pages, 2020. 